\title{
African trypanosomiasis: New insights for disease control
}

\begin{abstract}
African trypanosomes continue to present major public and livestock health problems in subSaharan Africa, typically in rural communities with limited access to diagnosis and treatment. Over the past 50 years, a tractable set of laboratory models for this infection has led to an appreciation of the immunological, cellular and molecular subtleties of trypanosomes, and in particular to their system of antigenic variation that is vital to the survival of these parasites in the host blood stream. Yet as the papers in this volume attest, there are still huge challenges to be met at both the basic and applied biological levels in order that the diseases caused by the African trypanosomes can be effectively diagnosed, controlled and treated.
\end{abstract}

The first stage in any programme to control human African trypanosomiasis is diagnosis, and this is made difficult by a combination of an ambiguous aetiology together with low parasitaemia. Magdalena Radwanska reviews progress in this area, including new techniques for detection of parasites using novel immunological (nanobodies) and molecular (LAMP) approaches that may be appropriate for deployment in the field in the medium term. Once a positive diagnosis is obtained, drugs are needed. As Christian Burri explains, the current armamentarium of drugs is 50 years old, with problems associated with treatment failure, adverse-reactions (particularly with melarsoprol) and continued availability. While a number of new drug leads are being pursued, the failure of the phase 3 trial of parfuramidine (DB289) has led to a greater circumspection in relation to the new drug leads currently under investigation. Meanwhile considerable progress has been made in optimisation of treatment regimens with melarsoprol and novel drug combinations such as nifurtimoxeflornithine combination treatment (NECT). A critical aspect of the pathogenesis (and choice of treatment) for human African trypanosomiasis is the invasion of the brain by the parasites. Jean Rodgers gives a comprehensive review of trypanosomiasis in the brain, including neuropathology and potential new agents that may be co-administered during treatment to minimise the serious consequences of reactive encephalitis. In recent years, there has been renewed interest in the pathogenesis of HAT in field studies, and recognition that the currently available laboratory models of infection may not reflect the diversity in progression and virulence of this disease. Lorna MacLean and Jeremy Sternberg give an account of the diversity of both $T . b$. rhodesiense and T. b. gambiense infections, including severe, mild and possibly asymptomatic cases. These naturally occurring virulence variants are potentially keys to unlock the molecular mechanisms underlying virulence in HAT and therefore provide new drug targets.

The special issue then turns its attention to recent developments in experimental work in African trypanosomiaisis with a direct bearing on disease control. Antigenic variation has long been viewed as an insurmountable barrier to the development of a vaccine against African trypansome infections. Angela Schwede and Mark Carrington review this process and detail our current knowledge of the plasma membrane and variant and invariant surface glycoproteins. The invariant surface glycoproteins have been proposed as potential vaccine candidates, and Stefan Magez et al. review these as well as potential anti-disease vaccine strategies. In both cases there are serious difficulties due to a failure of $\mathrm{B}$ cell memory in trypanosome-infected hosts. One factor in this process is likely to be a serious lesion in B cell development caused in trypansome infection, reviewed here by Sam Black and colleagues. Finally, John Mansfield and colleagues describe how African trypanosomes, as well as impacting on lymphopoiesis, may also modulate the activation of macrophages and dendritic cells in a manner that is profoundly sensitive to the timing of exposure to GPI and other VSG substituents. This process is a significant determinant of the subsequent virulence phenotype of infection.

The three guest editors would like to thank all the authors for their contributions to this timely volume and Dr Les Chappell, the co-ordinating editor, for his advice and encouragement.

Jeremy M. Sternberg, Samuel J. Black and Stefan Magez September 2010 